\title{
LA NOCIÓN DE BIENESTAR SUBJETIVO Y EL CONCEPTO DE DERECHO. A PROPÓSITO DEL VÍNCULO ENTRE NORMAS JURÍDICAS Y FELICIDAD HUMANA
}

\section{The Notion of Subjective Well- BEING AND THE CONCEPT OF LAW. ON THE LINK BETWEEN LEGAL Standards and Human Happiness}

A NOÇÃO DE BEM-ESTAR SUBJETIVO E O CONCEITO DE DIREITO. A PROPÓSITO DO VÍNCULO ENTRE NORMAS JURÍDICAS E FELICIDADE HUMANA

RAÚL MADRID-RAMÍREZ*

orcid.org/0000-0003-4592-6985. Pontificia Universidad Católica de Chile. Chile. rmadrid@uc.cl.

RECIBIDO: 16 DE NOVIEMBRE DE 2016. ENVÍO A PARES: 13 DE DICIEMBRE DE 2016 APROBADO POR PARES: 13 DE MARZO DE 2017. ACEPTADO: 16 DE MARZO DE 2017

DOI: $10.5294 / D I K A .2017 .26 .1 .3$

PARA CITAR ESTE ARTÍCULO / TO REFERENCE THIS ARTICLE / PARA CITAR ESTE ARTIGO RAÚL MADRID-RAMÍREZ, "LA NOCIÓN DE BIENESTAR SUBJETIVO Y EL CONCEPTO DE DE- 


\section{RESUMEN}

El objetivo de este trabajo es analizar la idea contemporánea del bienestar subjetivo (subjective well being) como equivalente de la noción clásica de felicidad, así como los parámetros que de este bienestar subjetivo se derivan para formular una relación con el derecho, entendido como el conjunto de prescripciones normativas vigentes en la comunidad. Con este propósito, se revisa sumariamente la noción tradicional aristotélica de felicidad, para comparar las diferencias entre esta y la idea de bienestar subjetivo, cuyo desarrollo ocupa el cuerpo del trabajo. Las conclusiones señalan la relación con los efectos para la comprensión del derecho que se derivan de las metodologías relativas a la noción de subjective well being.

\section{PALABRAS CLAVE}

Felicidad; derecho subjetivo; bienestar subjetivo; calidad de vida; preferencias. 


\section{ABSTRACT}

The aim of this work is to analyze the contemporary idea of subjective well-being as equivalent to the classical notion of happiness. The parameters that derive from this subjective well-being are examined as well, in an effort to formulate a relationship with the law, which is understood as the entire body of normative prescriptions in force in a community. With that end in mind, the traditional Aristotelian notion of happiness is summarized to compare the differences between it and the idea of subjective well-being, the development of which occupies the body of this work. The conclusions point to the relationship with the effects - for understanding the law - that are derived from the methodologies related to the notion of subjective well-being.

\section{KEYWORDS}

Happiness; subjective right; subjective well-being; quality of life; preferences. 


\section{RESUMO}

O objetivo deste trabalho é analisar a ideia contemporânea do bem-estar subjetivo (subjective well being) como equivalente da noção clássica de felicidade bem como os parâmetros que, desse bem-estar subjetivo, são derivados para formar uma relação com o direito, entendido como o conjunto de prescrições normativas vigentes na comunidade. Com esse propósito, revisa-se sumariamente a noção tradicional aristotélica de felicidade para comparar as diferenças entre esta e a ideia de bem-estar subjetivo, cujo desenvolvimento ocupa o corpo do trabalho. As conclusões indicam a relação com os efeitos para a compreensão do direito que são derivados das metodologias relativas à noção de subjective well being.

\section{PALAVRAS-CHAVE}

Bem-estar subjetivo; direito subjetivo; felicidade; preferências; qualidade de vida. 
SUMARIO: 1. LA FELICIDAD Y EL DERECHO; 2. LA FELICIDAD COMO VIDA VIRTUOSA Y SU DESARTICULACIÓN HISTÓRICA; 3. LA FELICIDAD COMO EL MAYOR BIENESTAR SUBJETIVO; 3. 1. EL CRITERIO DE LA “PREFERENCIA”; 3.2. EL CRITERIO DEL SUBJECTIVE WELL BEING; 4. El DERECho y EL bienestar subjetivo; Bibliografía.

\section{LA FELICIDAD Y EL DERECHO}

En principio, las ideas de "felicidad" y "derecho" no parecen vincularse directa o inmediatamente, sino de un modo secundario, oblicuo o incluso artificial. Si se efectuara el ejercicio de preguntar a un ciudadano promedio de cualquier comunidad occidental por el significado de estas dos nociones, respondería casi con total probabilidad que la felicidad consiste en tener salud, familia y determinada posición económica que le permita vivir a su gusto. El derecho, por su parte, sería identificado muy posiblemente con el conjunto de leyes que rigen el país donde reside, pero sin vínculo visible con la felicidad privada, salvo quizás permitir el desarrollo y la actualización de las expectativas individuales.

Solo en un segundo momento, el ciudadano en cuestión llegaría a establecer una relación con algún fundamento entre los dos conceptos que nos ocupan, pero dicha relación no surgiria de modo espontáneo, sino que tendría que ser dirigida, causada o formulada por el interrogador, para que saliera a la luz. Al parecer, el vinculo entre ambas nociones dista mucho de ser evidente para quienes no se dedican a analizar la naturaleza o esencia del derecho, salvo, como se apuntaba, en la vaga idea del derecho como articulador de las felicidades individuales.

En realidad, tanto el derecho como la felicidad son una preocupación constante en las sociedades contemporáneas, aunque por vías separadas. Se ocupan del primero para formular una cadena de exigencias al Estado y a los particulares, y de la segunda, porque, como dice Russell, los seres humanos en el mundo contemporáneo deberían ser felices, pero no lo son, al menos en la gran mayoría de los casos. ${ }^{1}$ De hecho - continúa el autor de Los principios de las matemáticas-, la gente parece estar lejos de alcanzar una sensación de felicidad, pues de observarla se deduce que experimentan ansiedad, exceso de concentración, falta de interés por todo lo que no sea la lucha cotidiana, incapacidad para divertirse, falta de consideración hacia el prójimo. ${ }^{2}$ A esta sensación de infelicidad colabora el "encuadramiento" (framing) que hacen los medios de comunicación de algunos aspectos específicos del bienestar humano, que son promocionados por estos como los elementos constitutivos y necesarios para ser feliz, en circunstancia de que suelen no estar al alcance de un elevado número de la población: el éxito, la belleza fisica, el lujo y los placeres concupiscibles de alta gama, lo que produce un hiato entre la situación vital real de las personas comunes y este estándar artificial del mensaje mediático, ${ }^{3}$ al mismo tiempo que orienta en un sentido determinado

Bertrand Russell, La conquista de la felicidad, Barcelona, Debolsillo, 2012, p. 21.

Ibid., p. 22.

Jean BAudrillard, La sociedad de consumo, Madrid, Siglo XII, 2009, pp. 113 ss.

DÍKAION - ISSN 0120-8942 - EISSN 2027-5366 / PP. 31-52 
la percepción común de lo que constituye materialmente el ser feliz. Esto resulta muy importante para la determinación contemporánea de la idea de felicidad, por cuanto hay un alto interés en comparar, desde una perspectiva sociológica o empírica, el contenido de lo que hace felices a los diversos individuos y grupos, ${ }^{4}$ pero también en imitar sus paradigmas, de tal modo que produce en ellos frustración (infelicidad) al no poder conseguirlo.

Tras la apariencia de constituir dos tópicos totalmente separados, que no se intersectan, las nociones de felicidad y derecho se encuentran, en realidad, sólidamente entrelazadas, como esperamos exponer suficientemente a lo largo de este trabajo. El derecho es un medio que auxilia a los hombres para alcanzar la felicidad; no porque se la proporcione él directa o materialmente, en su calidad de estructura normativa -imperativa, permisiva o prohibitiva-, sino porque constituye obligaciones positivas destinadas a la realización de algunos aspectos del bien personal, en dirección al bien común, y porque obra además como una especie de condición de posibilidad de la felicidad subjetiva e individual, al generar espacios institucionales que avalen el desarrollo de la vida y de la personalidad. La inclinación a la felicidad, por su parte, es universal en todos los hombres, y se configura como una aspiración común, que remite a la comunidad política como lugar en el que el ser humano alcanza su plenitud específica.

La tesis precedente, es decir, que una comprensión profunda del fenómeno jurídico en toda su extensión exige una comprensión de lo que es el hombre y de qué lo conduce hacia la vida buena, ha sido enfocada de, al menos, dos maneras a lo largo del pensamiento occidental: 1) tomando como punto de partida la idea de que la felicidad consiste en una actividad contemplativa, universal para todos los seres racionales que comparten una misma naturaleza; y 2) aproximándose a la felicidad como equivalente al bienestar subjetivo de cada persona, considerada en cuanto conciencia individual. Estas dos propuestas dan origen a diferentes ideas sobre la relación entre ambos conceptos, y configuran visiones distintas sobre el sistema jurídico, su naturaleza, finalidad y limites. El objetivo de este trabajo es formular algunas de las consecuencias para la comprensión del derecho que brotan desde este segundo paradigma, es decir, el modelo de derecho que es compatible con la felicidad configurada como bienestar subjetivo. Para llevar a cabo este propósito haremos primero una sumaria referencia a la concepción clásica de la felicidad, para analizar a continuación, en el contraste con el paradigma precedente, las características del nuevo modelo.

\section{LA FELICIDAD COMO VIDA VIRTUOSA Y SU DESARTICULACIÓN HISTÓRICA}

Dice Aristóteles que la felicidad es una cierta actividad del alma según la virtud perfecta. ${ }^{5}$ Ha dicho en otro lugar que todo el que es capaz de vivir según la pro-

4 Richard Layard, Happiness: Lessons from a New Science, New York, Penguin, 2011, pp. 87 ss.

5 ARIstóteles, Ética a Nicómaco, I, 13, 1102a5-7. 
pia decisión deliberada, ha puesto un cierto objetivo del buen vivir, mirando hacia el cual realizará todas las actividades, ya que el no ordenar la vida hacia un determinado fin es signo de gran insensatez. ${ }^{6}$ De este modo, consagra una ética teleológica en que cada acción, incluso la más insignificante, tiene sentido en el horizonte del fin último.

Pero este fin último ha de ser una actividad del alma, según la virtud perfecta de las facultades superiores y propias del hombre: virtud intelectual y virtud moral. Se trata de un fin perfecto y autosuficiente de la totalidad de las actividades que configuran la vida práctica. ${ }^{7}$ Esta actividad es identificada por Aristóteles con la función esencial (ergon) del ser humano en cuanto tal. ${ }^{8}$ La felicidad es la actividad propia del alma racional, según su virtud propia; esta actividad debe ser ejercida a lo largo de una vida completa. ${ }^{9}$ Ahora bien, Aristóteles admite, en principio, dos formas de vida feliz: la vida política, que se orienta al pleno ejercicio de las virtudes éticas en la razón práctica y su especial tipo de prudencia (phrónesis), y la vida contemplativa o teórica, centrada en el ejercicio pleno de las virtudes intelectuales y el uso especulativo de la razón. A esta última, sin embargo, la considera la forma suprema de la vida feliz. ${ }^{10}$

Ahora bien, la vida contemplativa, para poder ejercerse, requiere de un conjunto de condiciones que supongan la existencia cooperadora de terceros, es decir, de lo que se denomina tradicionalmente como "sociedad civil". La razón primaria de esta necesidad de colaboración se encuentra en que los hombres son individuos de la especie humana, de tal forma que esta solo se verifica en la sociedad de todos los humanos, pasados, presentes y futuros. Este es el bien común de la especie: el conjunto de perfecciones contenidas dentro de la esencia humana. Así pues, la perfección del individuo está en la perfección de su especie o esencia; es lo que se llama el "bien común específico".

En la medida en que la idea de una comunidad o sociedad civil mundial no es posible, por varias razones que no es del caso tratar aquí, ${ }^{11}$ los hombres se agrupan de hecho y por naturaleza en diversas sociedades concretas y accidentales, vinculadas a su temporalidad y espacialidad. Nace así la idea del bien común político: el bien de esa comunidad singular, hic et nunc, que Santo Tomás define como "el bien de la persona humana en sociedad”. Es este bien común el que puede llegar a permitir que algunos de sus miembros se dediquen a la vida contemplativa, sin perjuicio de que una mayoría opte por la virtud a través de la vida práctica.

6 ARISTóteles, Ética a Eudemo, I, 2, 1214b6-11.

7 Alejandro VIGo, La concepción aristotélica de la felicidad, Santiago, Universidad de Los Andes, 1997, p. 52.

8 ARISTóteles Ética a Nicómaco, 1098a5-6.

$9 \quad$ Ibid., I, cap. 3 y 8-11.

10 Ibid., X, caps. 6-9.

11 En una comunidad mundial, por ejemplo, no sería posible ejercer el efecto directivo del buen gobierno hacia el Bien Común, en virtud de las dimensiones de esa eventual comunidad mundial. 
La ley dirige y orienta la conducta de los hombres hacia el bien común, que es su propio bien, y como tal, colabora en la consecución de la virtud perfecta. La norma jurídica es expresión de la razón, y causa el derecho de cada uno en orden a que cada individuo alcance su mayor perfección posible, ya sea a través de la protección de los bienes naturales del hombre, como mediante la producción de los ámbitos sociales para el ejercicio de la libertad racional. De este modo, existe una indudable relación entre el derecho considerado como el conjunto de normas que rigen una sociedad, y la felicidad. La norma, al ser racional, se orienta a la mayor perfección de los individuos, permitiéndoles establecer un orden social que proteja los bienes naturales de la especie, y estimule la libertad a través de su protección jurídica.

En la tesis de raíz aristotélica el derecho, en su dimensión normativa, aparece como el instrumento mediante el cual se ponen las condiciones que hacen posible llegar al bien común, que es el bien personal del sujeto en sociedad. La vida virtuosa - que no es otra que la vida feliz - se puede alcanzar a través del cumplimiento de los fines de la naturaleza y de la razón, es decir, por medio de sistemas normativos (la moral, el derecho) que establecen los requisitos de la acción y determinan lo suyo de cada uno, permitiendo al ciudadano exigir de los terceros el respeto de esos derechos, ya sea a través de una acción o de una omisión, en un clima de orden y de certeza que constituye una condición de posibilidad para el desempeño de las actividades más altas del espíritu.

Esta concepción finalista y esencialista de la felicidad, que se acaba de esbozar, pierde fuerza "popular" a lo largo del decurso histórico, por las mismas razones que la concepción metafísica de la realidad comienza a retroceder de la opinio communis a partir de la modernidad y su concepción de que no existen verdades indudables fuera del plano lógico-empírico. Si la felicidad, al igual que la moral, ya no puede ser predicada según el formato de proposiciones universales para todos los hombres (como parece deducirse de un número relevante de autores modernos y posmodernos), su determinación queda depositada en los intereses psicológicos subjetivos de los individuos o de grupos de individuos. La política y la moral pasan, entonces, de fundarse en los principios de la psicología filosófica - que contemplaba principios universales - a residir en los presupuestos de la psicología científica, la sociología y la estadística, que se limitan a describir hechos. Así, la afirmación de que para comprender el derecho es necesario comprender primero a las personas, se transforma en este nuevo modelo, no en una consideración que apela a principios universales constitutivos de la naturaleza o de las estructuras de la racionalidad humana — no en una antropología filosófica-, sino en una perspectiva psicosociológica: interesa para determinar el contenido de las prescripciones y de la felicidad, como veremos, lo que los individuos hacen de hecho, y por qué lo hacen, no si existe una configuración universal de lo humano que pueda plantearse como fuente de rectitud en el comportamiento.

Una vez que el énfasis en la metodologia, propio de la llamada "época moderna”, consigue erradicar las concepciones metafísicas de la posición dominante 
en el debate filosófico occidental, las ciencias particulares comienzan paulatinamente a resentir la desestructuración sistemática de sus fundamentos, en un proceso progresivo de pérdida de identidad disciplinar y de atomización, lo que trae como consecuencia una fragmentación epistemológica, es decir, un estado o modelo de la ciencia en que no existe jerarquía entre las diferentes disciplinas. Esta condición se origina en la imposibilidad metodológica de concebir una ciencia del fundamento que estructure y dote de significado unitario al esquema general de la episteme.

El derecho no es ajeno a esta transformación. Hitos como la hipótesis Etiamsi daremus, de Grocio, muestran cómo el proceso de pérdida del fundamento trascendente da origen a una concepción puramente racionalista del derecho, encarnada por la llamada Escuela Moderna del Derecho Natural. Grocio sostenía, con objeto de probar que la ley natural estaba inscrita en el corazón de los hombres, que, aunque Dios no existiera, dicha ley seguiria estando en el ser humano. ${ }^{12}$ Con ello corta la referencia a la ley eterna, y abre paso al racionalismo jurídico en sentido moderno. Lo que hoy denominamos Ciencia del Derecho comienza a levantarse sobre estas bases, es decir, a partir de un progresivo distanciamiento de la idea del derecho natural de corte metafísico. Ello deriva en el reemplazo de dicha cátedra por la de Filosofia del Derecho. El último gran representante de esta línea de fundamentación es Kelsen, y su esfuerzo por abordar el fenómeno jurídico desde bases neokantianas. Kelsen buscaba liberar el ámbito jurídico de lo que él consideraba elementos extraños al mundo normativo - las nociones trascendentes en general: justicia, derecho de la naturaleza humana, etc.-, y se orientaba, a contrario sensu, a formular las bases de una ciencia jurídica formalmente impoluta de elementos ajenos a la especial perspectiva del derecho. Su trabajo, junto con el de Habermas - desde mediados del siglo XX-, constituye el último esfuerzo por generar una concepción del derecho sobre algún tipo de base universal, ya fuera la idea trascendental de la Grundnorm o la racionalidad comunicativa.

La posmodernidad, sin embargo, ejecuta el efecto inverso. Como deconstruye la fijeza de las identidades, discute también la peculiaridad epistemológica del derecho, evitando la primacía de la perspectiva jurídica en el análisis normativo, a través de la incorporación de otras ciencias, que son convocadas no en calidad de información relevante para la decisión de los operadores jurídicos, sino como parte de la producción normativa misma. Así, ciencias como la economía, la sociología o la psicología dejan de ser material consultivo para la configuración del enfoque propio del derecho, y pasan a desempeñar un papel relevante en la decisión jurídica y en la formación de la ley.

12 "Et haec quidem quae iam diximus, locum aliquem haberent etiamsi daremus, quod sine summo scelere dari nequit, non esse Deum, aut non curari ab eo negotia humana”, Hugo Grocio, "Prolegomena, 11", en Philip Christiaan Molhuysen, Hugonis Grotii. De iure belli ac pacis. Libri tres, in quibus ius naturae et gentium, item iuris publici praecipua explicantur: cum annotatis auctoris, Nueva Jersey, 2005. 


\section{LA FELICIDAD COMO EL MAYOR BIENESTAR SUBJETIVO}

A medida que el realismo filosófico comienza a perder posiciones en la escena académica, se produce una opacidad del concepto de "sujeto", que va perdiendo poco a poco su consistencia esencial hasta derivar en la idea del yo como un mero flujo de conciencia, propia de las posiciones posestructuralistas. ${ }^{13}$ Así, en lugar de configurar un individuo autoidéntico, el sujeto aparece de manera exclusiva a propósito de los actos particulares de interpretación, pero no necesariamente guarda una continuidad temporal consigo mismo. Por ello, las nuevas aproximaciones al concepto de felicidad tienden a analizar al yo como una realidad incomunicable, menos metafísica y más psicológica, que puede ser concebida solo a partir de sus actos. Por "realismo filosófico" entendemos aquí la posición opuesta a esta última, a saber, que el objeto del pensamiento es una cosa real exterior a la conciencia, dotada de esencia y existencia, que el entendimiento humano refleja, pero no crea en sus determinaciones esenciales. En esta línea de pensamiento, el sujeto es concebido como un ente sustancial, y no un mero devenir de la conciencia.

La concepción clásica de la felicidad, que antes trazamos de modo muy esquemático, extiende su influencia en el mundo occidental hasta que la propuesta de Bentham pasa a ocupar la posición dominante. ${ }^{14}$ La progresión conjunta de la pérdida de sustancia metafísica, y el advenimiento del utilitarismo en la concepción de la felicidad llevan a reemplazar la concepción universal de la vida virtuosa, contemplativa o práctica, por la noción de "bienestar subjetivo" (subjective well being): estados subjetivos de placer y satisfacción, en cuyo ámbito la proposición "para comprender el derecho es necesario previamente comprender a las personas" tiene un contenido muy distinto del propuesto en la versión clásica de la persona como sujeto sustantivo y personal: en el nuevo contexto, "comprender a las personas" no indica formular los principios universales aplicables a la esencia ni a la acción humana en general, sino que se relaciona con el análisis empírico del comportamiento humano de hecho.

Siguiendo este camino trazado por el empirismo, desde mediados del siglo XX los juristas han dedicado parte relevante de su tiempo a analizar el comportamiento fáctico de los individuos que integran las comunidades politicas, lo que implica un movimiento disciplinar desde la filosofia, como ciencia de los principios universales que apunta a las verdades esenciales e inmutables, hacia disciplinas como la psicologia y la sociologia, en cuanto ciencias del dato mental o social que se orientan más bien a la verdad contingente, entendida desde el prisma exclusivo de la verificación. Así pues, la "felicidad" pasa a configurarse, a partir de esta varia-

13 “Una definición del hombre en términos de 'mortalidad': solo la finitud temporal de la existencia, el efectivo sucederse de las generaciones, y por tanto la muerte entendida no solo "ontológicamente", sino también "ónticamente" (en la terminologia de Heidegger), funda la posibilidad de la historia como transmisión de mensajes, como acontecimiento no accidental, sino ontológicamente relevante, de la alternancia de las interpretaciones”, Gianni VAтtimo, Más allá del sujeto, Barcelona, Paidós, 1992, p. 8.

14 Martha Nussbaum, "Who is the happy warrior? Philosophy poses questions to psychology", en Eric Posner y Cass Sunstein (editores), Law and Happiness, Chicago, The University of Chicago Press, 2010, p. 88. 
ción de paradigma, como los contenidos subjetivos que contentan o entristecen a individuos concretos, y no la vocación del alma a la vida buena, como ocurrió de un modo más o menos consensuado hasta el siglo XVIII.

Este traslado de la cuestión teórica a las ciencias lógico-empíricas que acabamos de describir no acontece exclusivamente, desde luego, en el ámbito de la filosofia pura, sino también en las áreas regionales del pensamiento. En el caso del derecho, el modelo de la filosofia del derecho (Rechtsphilosophie), que supone una reflexión sobre el fundamento $u$ origen radical del fenómeno jurídico, de donde deriva su regularidad y su universalidad, ${ }^{15}$ es reemplazado como posición dominante por la Teoría del Derecho (Legal Theory), que constituye más bien una reflexión sobre la ratio metódica de la disciplina, con un fuerte contenido interdisciplinar, sin que ello signifique o importe una primacía hipotética de la perspectiva jurídica. En otros términos: en la Teoría del Derecho la justificación técnica de la argumentación jurídica incorpora otros hábitos científicos, como la psicología y la sociología, no solo como fuentes de referencia, sino en un plano de igualdad formal con la perspectiva jurídica. ${ }^{16}$ Desde esta perspectiva, es incapaz de distinguir entre la ciencia principal y las auxiliares.

Producido pues este deslizamiento desde la felicidad como una noción metafísica, y, por tanto, relacionada con la esencia abstracta del hombre, hacia un paradigma metodológico descriptivo — como el que se encuentra detrás de la idea de subjective well being - pueden advertirse dos criterios históricamente sucesivos que reclaman la identificación y objetivación del mencionado bienestar subjetivo. Hablaremos de ellos por separado.

\subsection{El criterio de la "preferencia"}

El primer criterio científico que se ha utilizado para intentar medir el grado de bienestar o felicidad de los ciudadanos proviene de la economia. La creciente importancia de las ciencias económicas se debe —entre otras causas-, al predominio de un paradigma metodológico neomarxista, cuya influencia se encuentra vigente hasta nuestros días. Este modelo opera como catalizador de la reducción de los criterios sociales a una dialéctica de acumulación del poder en términos económicos, lo que contribuye a poner en primer plano el análisis crematístico.

El punto de partida económico - para el tema que aquí nos interesa- es que los individuos actúan racionalmente en la consecución de sus intereses. Esta búsqueda de los propios intereses se encuentra limitada por la información que posee el sujeto, por su capacidad para procesarla de cara a los objetivos que se ha planteado, y por el influjo de sus emociones, lo cual lleva a que en muchas ocasiones

15 "La ontología jurídica indica las investigaciones filosóficas sobre la esencia del Derecho, que quieren exponer las características fundamentales de todo sistema jurídico, y no solo de un ordenamiento jurídico determinado, históricamente dado", Otta WeINBERGER, Norm und Institution. Eine Einführung in die Theorie des Rechts, Maguncia, Manz Verlag Viena, 1988, p. 16.

16 Raúl MADRID, "Situación de la Teoría del Derecho en el contexto de las ciencias fronterizas", en Seminarios de Filosofia 8 (1995), p. 241. 
sean incapaces de arribar a las metas propuestas. Estas metas son cuantitativas, medibles, siendo apreciadas por el agente en virtud de su utilidad. ${ }^{17}$ El grado en que las preferencias se alcanzan depende del presupuesto del que cada uno dispone, lo cual estará determinado, a su vez, tanto por sus ingresos como por el precio de los bienes. De ahí la especial atención que se presta al ingreso per capita. Desde el punto de vista jurídico, este análisis se enmarca dentro de la escuela conocida como Law \& Economics, una corriente propia de la Teoría del Derecho que se caracteriza por aplicar métodos propios de la economía a los conflictos jurídicos, intentando predecir desde bases económicas los efectos de las normas jurídicas. ${ }^{18}$

En esta dimensión, la felicidad queda definida por la capacidad de alcanzar sus intereses económicos, y la infelicidad, por el fracaso de este empeño. De hecho, desde hace casi cuarenta años los académicos han intentado cuantificar el bienestar utilizando el análisis de costo / beneficio, buscando proponer un criterio objetivo que permita identificar qué hace feliz a las personas. El argumento, en terminologia de Pareto, se puede fundar en utilidades ordinales, que imponen una carga menor de información al agente decisor que las utilidades cardinales, lo que orientaría de modo apropiado la formulación de las leyes y las políticas públicas. ${ }^{19} \mathrm{~A}$ su vez, las críticas a este planteamiento se refieren básicamente a: a) las dificultades metodológicas para utilizar las preferencias declaradas por los ciudadanos como representativas del bienestar, y b) las limitaciones de la aproximación que resulta del análisis costo / beneficio a la hora de definir el valor de la vida. ${ }^{20}$

A pesar de esta aproximación crítica, el análisis de costo / beneficio se ha seguido utilizando de modo casi universal, por cuanto ningún método alternativo ha alcanzado el valor de un acuerdo doctrinal pacífico. Si bien es verdad que el modelo economicista para explicar el bienestar de los sujetos humanos ha dominado la escena por un largo tiempo, no es menos verdadero que el esquema de la felicidad/placer ha estado siempre rondando en los márgenes, cuestionándolo e interrogándolo permanentemente. El ejemplo más famoso de tal actitud es la llamada "paradoja de Easterlin" (1973), mediante la cual se pone en tela de juicio la teoría económica tradicional, que supone que mientras mayor es el nivel de ingresos, mayor será el nivel de felicidad. Diversos estudios contemporáneos han demostrado, en la misma línea, que el efecto del dinero en la felicidad individual es mucho menor de lo que se suponía. ${ }^{21}$

17 Irving Fisher, "Is "utility the most suitable term for the concept it is used to denote?", en American Economic Review 8 (1918), pp. 335-7.

18 Cfr. Ronald CoAse, The Problem of Social Cost, Virginia, The University of Virginia Press, 1960.

19 Vilfredo PAREto, Manuale di economía política, con una introduzione a la scienza sociale, Roma, Societa Editrice Libreria, 1906. Las utilidades ordinales, a diferencia de las cardinales, pueden inferirse, en principio, de la observación del comportamiento del consumidor. En otros términos: atender al deseo de pagar o aceptar el precio de la cosa querida permitiria formular las políticas públicas y las prescripciones normativas.

20 John Bronsteen, Christopher Buccafusco y Johnathan Masur, Happiness and the Law, Chicago, University of Chicago Press, 2014, pp. 59 ss.

21 Ed Diener et al., "The relatioship between income and subjective well being: Relative or absolute?", en Social Indicators Research 195 (1993), p. 221. 
En la actualidad, si bien el método económico continúa vigente en muchas esferas, no es menos verdadero que ha perdido la confianza que se depositaba en sus predicciones durante las décadas pasadas, y se le reconocen un conjunto significativo de falencias, relativas fundamentalmente: a) al uso de las preferencias declaradas como representativas del bienestar real, y b) a la forma en que define el valor de la vida, vinculada con los valores monetarios, que no son constantes a lo largo del tiempo, y resultan, en consecuencia, poco adecuados a la hora de retratar el bienestar. ${ }^{22} \mathrm{El}$ argumento es que, si bien el análisis costo/beneficio sucumbe ante estas criticas, el criterio del bienestar subjetivo no lo hace, porque el bienestar, a diferencia del dinero, es invariable en el tiempo. Probablemente, además, el nuevo paradigma tenga la virtud de considerar al ser humano desde una perspectiva más integral y menos materialista que la simple exposición de sus preferencias en el ámbito del gasto, aunque se encuentre basado también en información estadística. Veamos a continuación en qué consiste.

\subsection{El criterio del subjective well being}

En los últimos años, a partir de las objeciones a la explicación meramente económica del bienestar - pero también de la creciente participación de otras ciencias del área social y humana en el análisis - ha surgido el llamado happiness approach, que se apoya en encuestas en las que se solicita a las personas que califiquen su "felicidad" dentro de una escala. Este procedimiento evidentemente implica un giro hacia la psicologización del problema, por cuanto tal disciplina parece haber desarrollado mecanismos concretos para determinar qué haría más felices o infelices a los hombres, según sus circunstancias. El subjective well being pasa, así, a relacionarse no con indicadores objetivos - en el sentido de esenciales-, por muy reduccionistas que pudieran parecer algunos de ellos, sino con los informes y las referencias relativos a los "estados mentales" de la población, considerándolo un fenómeno psicológico cuya espina dorsal son los sentimientos y las sensaciones de placer y displacer, de satisfacción o de insatisfacción, capturados en un momento específico del tiempo y del espacio.

Esta perspectiva, a saber, que las políticas públicas y el derecho deben centrarse en la felicidad - entendida como el placer o bienestar-, y no en el orden de las preferencias (ni menos en un conjunto de bienes humanos naturales) es, por lo demás, muy poco novedosa en su contenido esencial, aunque reaparece ahora con fundamentos metodológicos distintos que apelan, como se dijo, a la imposibilidad de acudir a proposiciones universales e inmutables para configurar una noción de bien y felicidad sustantivamente común a todos los hombres. Ya Bentham había propuesto - por cierto, sin prestar atención alguna al debate filosófico precedente sobre el particular ${ }^{23}$ - una forma específica de utilitarismo que maximizaba el

22 John Bronsteen, Christopher Buccafusco y Johnathan Masur, Happiness and the Law, op. cit., pp. 59 ss.

23 Cfr. sobre este punto J. C. B. Gosling, Pleasure and Desire: The case of hedonism reviewed, Oxford, Oxford Clarendon Press, 1969. 
placer por sobre cualquier otro criterio, ${ }^{24}$ el cual define como una única sensación, que varía solo en las dimensiones cuantitativas de intensidad y duración, certeza y proximidad. ${ }^{25}$ La psicología contemporánea, por su parte, se inclina a compartir esta opinión puramente cuantitativa sobre la naturaleza del placer; ${ }^{26}$ Kahneman, por ejemplo - uno de los autores centrales en la concepción del subjective well being-, liga genealógicamente su concepto de "flujo hedónico" (hedonic flow) a la tesis benthamiana sobre el criterio del placer. ${ }^{27}$ En la actualidad, estos paradigmas hedonísticos se manifiestan en conceptos como hedonic psychology y hedonic adaptation; es decir, una psicología fundada específicamente sobre las sensaciones de placer / dolor, y el análisis de la capacidad del hombre para adaptarse tanto a lo placentero como a lo doloroso.

La cuestión es la siguiente: la perspectiva económica resulta demasiado rígida para determinar lo que las personas realmente quieren. El dinero no tiene un valor intrínseco desde el punto de vista de la felicidad, sino que su significado es relativo a los bienes y las condiciones vitales que puede conseguir. El problema que advierten los psicólogos es que en un número muy alto de casos, los individuos no son capaces de predecir lo que va a hacerlos felices. ${ }^{28}$ La gente se adapta fácilmente tanto a lo que cree que lo va a hacer feliz (por ejemplo, adquirir un auto elegante), como a lo que piensa que lo convertirá en infeliz (por ejemplo, perder un brazo). Este es el campo de la hedonic adaptation, antes mencionada, que puede tener una enorme influencia para la determinación del daño moral. ${ }^{29} \mathrm{El}$ efecto de este principio aplicado a la adaptación humana a la discapacidad física fue estudiado originalmente en un artículo de 1978, que tuvo gran repercusión en el análisis de la idea de felicidad para la determinación del derecho y las politicas públicas. ${ }^{30}$ En este texto se demostraba, básicamente, que ni los ganadores de la lotería se convertian en personas felices, ni aquellos que sufrian discapacidad por causa de un accidente eran tan desgraciados. Tal conclusión sugiere que las personas viven la vida como si fuera una "rueda de molino" (hedonic treadmill), en la que los

24 Jeremy Bentham, The Principles of Morals and Legislation, New York, Prometheus Books, cap. 1: "La naturaleza ha colocado a la especie humana bajo el gobierno de dos señores soberanos, el dolor y el placer [...] El principio de utilidad reconoce esa sujeción y la da por supuesta como fundamento del presente sistema, cuyo objetivo es edificar la fábrica de la felicidad con las manos de la razón y de la ley".

25 Ibid.

26 Martha Nussbaum, "Mill between Bentham and Aristotle”, en Daedalus 133 (2004), pp. 60-68.

27 Daniel Kahneman y Alan B. Kruger, "Developments in the measurement of subjective well being”, en Journal of Economic Perspectives 20 (2006), p. 4.

28 Daniel Kahneman y Angus Deaton, "High income improves evaluation of life but not emotional well being”, en Proceedings of the National Academy of Science, 107, 38 (2010), p. 16489. Es lo que se denomina "predicción afectiva”. Cfr. Timothy D. Wilson y Daniel T. Gilbert, "Affective forecasting”, en Advances in Experimental Social Psychology 35 (2003), pp. 345-411.

29 Por ejemplo: parece en principio más grave perder una pierna que padecer de tortícolis permanente, suponiendo que ambas fueran fruto de un mismo hecho (como un accidente). Sin embargo, con el paso del tiempo, la pérdida de un miembro es aceptada, y comienza un periodo de recuperación ascendente, mientras que la tortícolis puede afectar de un modo significativamente grave el desempeño cotidiano de las funciones y los aspectos normales de la vida, ¿cuál de los dos requiere una mayor indemnización?

30 Philip BRICKMAN et al., "Lottery winners and accident victims: Is happiness relative", en Journal of Personality and Social Psychology 36 (1978), pp. 917-27. 
acontecimientos malos o buenos producen cambios momentáneos en el bienestar subjetivo, desde los que se regresa con cierta rapidez a un punto de equilibrio. ${ }^{31}$ Semejante resultado recuerda la idea aristotélica de que tanto la fortuna como la desventura son adventicias. Esta semejanza no pasa de ser simplemente material, por cuanto el fundamento del Estagirita se relacionaba más bien con los principios naturales, de cuyo ejercicio brotaba la vida buena.

El objetivo de este modelo de análisis de la felicidad humana, lejos de apreciar comportamientos esenciales, o variables económicas, como en el método del costo / beneficio, aspira a presentar un análisis en el que sea posible apreciar en qué consiste la experiencia subjetiva del mal o daño sufrido por un individuo. Esto es lo que se denomina propiamente subjective well being, concepto que apunta al sujeto entendido en relación con una experiencia concreta, sin que el valor apreciativo de dicha experiencia singular pueda ser universalizado respecto de otras experiencias similares. En otros términos, el argumento se formula estadísticamente, no por su valor abstracto ni por sus variables de interés económico.

El concepto —objeto del área disciplinar conocida como psicología positiva— apunta al modo en que la gente experimenta la calidad de su vida, y comprende tanto factores cognitivos como emocionales, relativos a la satisfacción, los afectos positivos y negativos, y la felicidad en general, cuestión que se encuentra fuertemente vinculada a las características personales de cada cual. Se trata, en consecuencia, de una noción más amplia que la felicidad, ${ }^{32}$ pero que la reemplaza desde el punto de vista del objetivo de los individuos en sociedad. La felicidad como tal resulta difícil de medir, y, consagrada la necesidad de un criterio objetivo que tome el lugar de los postulados esencialistas, el concepto de subjective well being aparece como susceptible de aceptar valores de medición compatibles con el escenario metodológico. Como se anunciaba, el método para hacer cuantitativo este bienestar consiste en interrogar a las personas que padecen dichas experiencias, confiando en la información que proporcionan sobre su bienestar individual. El bienestar de los sujetos se estructura en una escala de 10 a 0 , en la que el primero indica la "felicidad perfecta", y el segundo su contrario. La principal pregunta es: "tomando los diversos aspectos, ¿qué tan satisfecho se encuentra actualmente usted con su vida considerada como un todo (your life as a whole)?”33

Se estima que la medición debe ser: a) conceptualmente apropiada; b) válida, y c) empíricamente útil. ${ }^{34}$

a) Una medición conceptualmente apropiada es, para estos autores, la que atiende a lo que resulta bueno para el individuo concreto; no la que intenta

31 John Bronsteen, Christopher Buccafusco y Johnathan Masur, Happiness and the Law, op. cit., p. 17.

32 Ibid., p. 133.

33 Daniel Kahneman y Alan B. Kruger, "Developments in the measurement of subjective well being”, en Journal of Economic Perspectives 20 (2006), p. 7.

34 Paul Dolan y Tessa PEASGOod, "Measuring well being for public policy: Preferences or experiences?, en Eric Posner y Cass Sunstein (editores), Law and Happiness, Chicago The University of Chicago Press, 2010, pp. 8 ss. 
determinar qué es la vida buena (como ocurría en el modelo clásico). Y dentro de lo bueno para el individuo concreto, lo que interesará a los legisladores es solo el aspecto relativo a la materia sobre la que se quiere legislar, lo que supone excluir todos aspectos del bienestar que no sean relevantes para dicha proyección normativa. En este punto, cabe formular una diferencia entre "vida buena" y "vida buena de un individuo concreto", según se deduce de la idea de bienestar subjetivo: el primero incluye bienes y valores que se encontrarian más allá de lo que resulta apropiado para el sujeto concreto-verificable, como la moral, los bienes espirituales, la belleza, etc. Así, la noción de bienestar subjetivo que cabe ser utilizada para efectos del derecho sería más bien la que se refiere a lo que parece adecuado aquí y ahora para el sujeto de carne y hueso. ${ }^{35}$ Esto es muy significativo para la caracterización de la teoría del subjective well being: su contenido abarcaría solo los aspectos medibles o cuantificables empíricamente de las sensaciones o los sentimientos de placer / dolor. Aunque se sostenga que los aspectos no considerados para la configuración del bienestar siguen presentes en la mente de los gobernantes y legisladores (la moral, etc.), estos deben ser expulsados de la medida del bienestar, para efectos de la transparencia y la claridad.

En esta idea parece advertirse, por tanto, una opción que busca reducir toda verdad en la verificación: el razonamiento es que, puesto que dichas categorias no son empiricas, entonces carecen de transparencia y claridad. La noción de subjective well being, por tanto, debe configurarse por fuerza como una construcción empírica, que incluya todos los atributos materiales que puedan hacer mejor la vida de cada uno, con excepción de lo ilícito o lo socialmente ilegitimo.

b) Para que sea útil al derecho y a las politicas públicas, una medición debe ser también "válida", es decir, debe medir lo que busca medir, aunque se reconoce que resulta imposible lograr esta determinación perfecta, en la medida en que no se cuenta con un estándar inmutable del bienestar subjetivo. Aun así, la medición debiera mostrar cómo las posibilidades del bienestar subjetivo de un sujeto cambian en el tiempo, y se comparan con el de otros.

c) Finalmente, la medición debe ser empíricamente eficiente, lo que indica que debe configurarse como una evaluación carente de prejuicios, y que al mismo tiempo no sea proclive a elementos teóricos o abstractos considerados como irrelevantes, por cuanto ambos elementos la pueden convertir en impracticable (ineficiente). Es preciso que se trate de una medición práctica, es decir, sencilla y no demasiado costosa de ejecutar.

El concepto de bienestar subjetivo puede ser objeto de mediciones empíricas con objeto de proponer criterios operativamente objetivos de análisis. No es lo mismo, sin embargo, hablar de la subjetividad como lo único que queda después de la supresión de los parámetros de universalidad esencial, que afirmar la subjetividad como un instante autopoiético en el flujo de la conciencia. En otros términos, hay una diferencia filosófica abismal entre aislar la idea misma de conciencia subjeti-

35 Ibid., p. 11.

DÍKAION - ISSN 0120-8942 - EISSN 2027-5366 
va, en términos abstractos, y concebirla como una especie de pantalla en la que la realidad se configura y desarma a su arbitrio, sin significados permanentes. Esto último remite a concepciones mucho más radicales del yo, y convierte en espurios todos los intentos de dotar de algún grado de objetividad la sensibilidad sobre el bienestar o el malestar. Desde este modelo, que es, podríamos decir, el último estadio en el caminar de la idea de felicidad, se precipitan consecuencias que convierten al sujeto en una realidad incomunicable, impredecible y en permanente cambio. De todo ello se configuran resultados para el derecho.

\section{EL DERECHO Y EL BIENESTAR SUBJETIVO}

Hasta aquí la descripción del bienestar subjetivo, que ha reemplazado tanto a la noción de felicidad como al análisis de las preferencias. ¿Cuáles son los efectos para la concepción del Derecho que provienen de la aplicación de este modelo? Como conclusión general, hay que decir que la observación del comportamiento de los ciudadanos, erigido en el único método para descifrar la idea de felicidad, satisfacción o bienestar de los individuos en sociedad, lleva a que las nociones fundacionales sobre el derecho que ha utilizado la tradición jurídica sean puestas en cuestión.

4.1. El bienestar subjetivo se encuentra en directa relación con la calidad de vida, que se configura como un concepto variable y meramente descriptivo. Según se recordará, la pregunta central es por la vida "como un todo", lo que entraña una profunda ambigüedad sobre el contenido de la pregunta. Se interroga sobre un cierto estado de satisfacción subjetivo, pero también constituye una invitación a reflexionar sobre la propia vida, lo que puede ir acompañado o no de sensaciones o sentimientos de satisfacción. Para los psicólogos, esta ambigüedad no se presenta como especialmente significativa, ${ }^{36}$ pero sí lo es para el ámbito del derecho.

La definición de la calidad de vida es doctrinalmente compleja, y oscila entre argumentos que intentan guardar en ella una cierta objetividad, y aquellas propuestas que la entregan en mayor o menor medida a la determinación por parte de la conciencia individual. ${ }^{37}$ La doctrina ha identificado cuatro elementos que la compondrian: a) la calidad de las condiciones de vida; b) la satisfacción experimentada con dichas condiciones vitales; c) la combinación de componentes objetivos y subjetivos, es decir, definida como la calidad de las condiciones de vida de un individuo junto a la satisfacción que experimenta y, d) la combinación de las condiciones de vida y la satisfacción personal ponderadas por la escala de valores, aspiraciones y expectativas personales. ${ }^{38}$ Como puede verse en esta enumeración, y sin perjuicio de que se pueda esta-

36 Martha Nussbaum, "Who is the happy warrior? Philosophy poses questions to psychology", op. cit., p. 87.

37 Una buena enumeración de estas posiciones en Alfonso Urzúa y Alejandra CAgueo-Urizar, "Calidad de vida: una revisión teórica del concepto", en Terapia Psicológica XXX, 1 (2012), p. 62.

38 David Felce y Jonathan Perry, "Quality of life: It's Definition and Measurement”, en Research in Developmental Disabilities 16, 1 (1995), pp. 51-74. 
blecer un mínimo objetivo de condiciones vinculadas con lo necesario para el desarrollo de las condiciones materiales y espirituales del hombre, la noción de calidad de vida posee un componente intrínsecamente subjetivo.

Esta naturaleza incomunicable, ya sea que aborde un enfoque de la vida completa en general, o constituya una especie de "fotografia" de esta en un cierto tiempo y espacio, adquiere una ambivalencia particularmente intensa si su contenido tiende a ser determinado exclusivamente con base en mecanismos psicológicos, como ocurre de hecho una vez que la psicología filosófica emprende su retirada de la escena disciplinar antropológica. Así, la pregunta por el concepto de derecho que brota de un sistema jurídico ordenado a la calidad de vida forzosamente describe un concepto de hombre que se configura por datos tomados de la experiencia individual.

La felicidad (o satisfacción, o calidad de vida) se convierte de este modo en el objeto final de todas las prescripciones normativas, y aparece como un derecho difuso, entendiendo por tal aquel que no está determinado a priori en su contenido, pero tampoco es determinable por parámetros de interpretación preexistentes y válidos. En otros términos: solo cabe ser determinado en cada acto por el sujeto, que acude a su subjetividad para encontrar las razones o causas de su satisfacción circunstancial. Esta consecuencia puede tener efectos devastadores para la certeza y eficacia del ordenamiento jurídico, y debiera ser objeto de un análisis detenido.

El efecto que hemos mencionado no se produce exclusivamente con el derecho a la felicidad, sino que afecta a todas aquellas exigencias jurídicas en las que resulta arduo separar, con el instrumental metodológico-disciplinar de la Ciencia del Derecho tradicional, los meros intereses de los derechos subjetivos.

4.2. Como consecuencia de lo anterior, el derecho aparece conectado de manera excluyente con la forma en que, en los hechos, los ciudadanos viven, se satisfacen y buscan su bienestar sensible / sentimental; sin embargo, no vinculado a principio general o abstracto alguno que indique una ratio universal de comportamiento moral por encima de las experiencias de satisfacción o desagrado. Las normas, por tanto, no tienen un contenido teleológico en sentido fuerte, sino que se miden exclusivamente por razón de su capacidad o eficacia para hacer más grata la vida de la población. Los hechos descritos por el comportamiento se aplican luego al derecho, el que se pone en práctica a su turno con objeto de afectar de modo positivo la satisfacción de los ciudadanos. Para esto, los legisladores deben contar con un instrumental que mida el efecto de los proyectos de ley en dicho bienestar.

4.3. Es interesante atender a las críticas $u$ objeciones que los propios defensores de esta idea del bienestar psicológico advierten, por cuanto arroja cierta luz sobre la manera en que conciben su relación con el derecho. La cuestión es determinar si la idea de bienestar subjetivo es mejor que otros 
procedimientos (como el análisis de preferencias) para evaluar la bondad o adecuación de las normas jurídicas. Esto dependerá de si es un sistema capaz de medir el bienestar "neto" de los ciudadanos. Y la respuesta es que falla en hacerlo, pero yerra menos que todos los otros métodos. La razón por la cual justifican esta debilidad metodológica es que "la vida humana es tan caóticamente complicada que se evade de los intentos de analizarla con completa exactitud y precisión”. ${ }^{39}$

Al tenor de estas explicaciones, parece posible arribar a la conclusión de que quienes sostienen esta tesis consideran la "complejidad" de la vida humana más bien como un defecto que debe ser subsanado, una especie de "error" de la naturaleza que la mente humana está obligada a corregir para disminuir y eliminar la incerteza, que acecha con la impureza de la duda. Así, alcanzar el bienestar neto se convierte en un ideal fuera de este mundo cotidiano, en el que la vida misma, como un conejo correteando en el parque, se "evade" del análisis preciso y tranquilizador.

Esta posición es exactamente opuesta a la que propone la idea clásica de la felicidad como actividad práctica o contemplativa. Para estos autores, la vida humana es también compleja, pero esta "complejidad" les parece propia de naturaleza la vida práctica — no un defecto que debe ser eliminado mediante la corrección del método-, y no constituiria, por tanto, un obstáculo para la determinación de la felicidad como vida virtuosa; antes bien, la vida virtuosa supondría la consideración integral del sujeto como un ente a la vez concreto (singular, material, complejo) y universal (espiritual, formal, simple), con plena transitividad entre ambos mundos para dar lugar al individuo real, cuya felicidad se encuentra situada a medio camino entre los dos ámbitos, y no solo en el puramente psicológico.

¿Qué efectos arroja esta diferencia de visión sobre el concepto de derecho y su función? Para los defensores de la idea de felicidad como actividad del espíritu (a), el derecho - las prescripciones normativas justas- constituye un medio para alcanzar los bienes propios en comunidad, y, por tanto, es en cierta forma causa eficiente de la felicidad, o de aspectos concretos de ella, por cuanto la "felicidad plena" viene a ser semejante a la sabiduria: nunca puede agotarse exhaustivamente en la condición humana. Luego el derecho tiene un significado trascendental: ayudar a los hombres a ser más perfectos en medio de la vida comunitaria, porque de esa perfección dependerá su grado de felicidad. La obligatoriedad de la norma tiene, en este sentido, un valor primario y significativamente moral.

Para los autores que consideran la felicidad como equivalente al bienestar subjetivo (b), se presentan una serie de problemas metodológicos que no se verificaban en el modelo anterior. La infinita variedad y multiplicidad de la vida humana resulta un defecto a la hora de alcanzar una idea abstracta de

39 John Bronsteen, Christopher Buccafusco y Johnathan Masur, Happiness and the Law, op. cit., p. 44. 
ella, desde la inducción. Los clásicos sí disponían de esta idea abstracta, pero obtenida desde una premisa metafísica, lo que les permitía encajar la vida práctica y toda su diversidad con fluidez. Este nuevo modelo, sin embargo, requiere de una prótesis: algo que pueda servir como una especie de paradigma o base axiomática desde la cual configurar el contenido del bienestar subjetivo. En este contexto, el derecho ya no puede buscar el bien universal de los hombres, considerado como un principio idéntico en cada ser humano concreto (esencia común), sino que debe esforzarse solo por facilitar la convivencia y aumentar la calidad de vida de los ciudadanos, por cuanto evita que se causen daño, y si se lo causan mutuamente, las normas jurídicas los obligan a restablecer el orden alterado, mediante una compensación específica prevista para el caso. ${ }^{40} \mathrm{El}$ derecho existiria, por tanto, para mejorar la calidad de vida o el bienestar subjetivo de los ciudadanos, no para conducir al bien común, ni menos para perfeccionar al ser humano en la consecución de sus virtudes más altas.

4.4. La reducción material que se opera en el concepto de felicidad al ser reemplazado por la idea del bienestar subjetivo, donde quedan fuera, como se indicara, los aspectos trascendentes del bienestar humano - el bien, la verdad, la belleza-, reduciéndose a las dimensiones empíricas y cuantificables, tiene un efecto en la concepción del Derecho, incluso si se sigue sosteniendo en abstracto que el sistema jurídico protege dichos bienes, por cuanto no los considera para efecto de las prescripciones normativas concretas. Este mecanismo trae como consecuencia una mutilación del derecho, en la medida en que es una mutilación de la idea de felicidad humana, es decir, de la antropología de base. Este corte puede ser entendido más bien como una reducción, que termina por producir, para efectos del sistema jurídico, una caricatura de lo humano que puede generar innumerables problemas de fondo, pero también metodológicos, por cuanto la producción normativa se orientará a un ser humano constreñido a sus aspectos más periféricos: aquellos que son determinables por la experiencia. La idea clásica de la norma jurídica que conduce al hombre hacia su fin integral, por tanto, desaparece.

Parece, en consecuencia, que existen razones para afirmar que el modelo del bienestar subjetivo con el que se analiza en la actualidad la felicidad de los ciudadanos es fruto de un psicologismo extremo, al que se ha orillado la reflexión sobre el ser humano después de que sucesivos virajes metodológicos declaran la imposibilidad de concebir un sujeto sustancial, que se proyecte en el tiempo como una conciencia autoidéntica. Esta psicologización de la realidad humana se vierte, cómo no, en todos los ámbitos de la personalidad y la acción del hombre, incluido el derecho, donde genera una serie de preguntas metodológicas, la mayor parte de ellas relacionadas con la subsistencia de los objetivos tradicionales que la Ciencia Jurídica ha asignado al ordenamiento. 


\section{BIBLIOGRAFÍA}

ARISTóteles, Ética a Eudemo, Madrid, Gredos, trad. Pallí Bonet, 2003.

Aristóteles, Ética a Nicómaco, Madrid, Centro de Estudios Constitucionales, trad. Julián Marías y María Araújo, 1994.

Baudrillard, Jean, La sociedad de consumo, Madrid, Siglo XII, 2009.

Bentham, Jeremy, The Principles of Morals and Legislation, Nueva York, Prometheus Books, 2012.

BobBio, Norberto, Prólogo, en Luigi FerRasoli, Derecho y razón. Teoría del garantismo penal, Madrid, Trotta, 1995.

BRICKMAN, Philip et al., "Lottery winners and accident victims: Is happiness relative", en Journal of Personality and Social Psychology 36 (1978), pp. 917-27.

Bronsteen, John, Buccafusco, Christopher y Masur, Johnathan, Happiness and the Law, Chicago, University of Chicago Press, 2014.

CoAse, Ronald, The Problem of Social Cost, Virginia, The University of Virginia Press, 1960.

Diener, Ed. et al., "The relatioship between income and subjective well being: Relative or Absolute?”, en Social Indicators Research 195 (1993), pp. 195-223.

Dolan, Paul y PEASGOOD, Tessa, "Measuring well being for public policy: Preferences or experiences?, en Eric Posner y Cass Sunstein (editores), Law and Happiness, Chicago The University of Chicago Press, 2010, pp. 5-31.

Felce, David y PerRY, Jonathan, "Quality of life: It's definition and measurement", en Research in Developmental Disabilities 16, 1 (1995), pp. 51-74.

FisHeR, Irving, "Is 'utility the most suitable term for the concept it is used to denote?", en American Economic Review 8 (1918), pp. 335-37.

Gosuing, J. C. B., Pleasure and desire: The case of hedonism reviewed, Oxford, Oxford Clarendon Press, 1969.

Grocio, Hugo, "De iure belli ac pacis. Libri tres, in quibus ius naturae et gentium, item iuris publici praecipua explicantur: cum annotatis auctoris", en Molhuysen, Philip Christiaan. Hugonis Grotii, New Jersey, 2005.

Kahneman, Daniel y Deaton, Angus, "High income improves evaluation of life but not emotional well being", en Proceedings of the National Academy of Science 107, 38 (2010) 16489-16493.

Kahneman, Daniel y Kruger, Alan B., "Developments in the measurement of subjective well being", en Journal of Economic Perspectives 20 (2006), pp. 3-24.

MADRID, Raúl, "Situación de la Teoría del Derecho en el contexto de las ciencias fronterizas", en Seminarios de Filosofia 8 (1995), pp. 223-241.

LAYARD, Richard, Happiness: Lessons from a New Science, New York, Penguin, 2011. 
Nussbaum, Martha, "Mill between Bentham and Aristotle”, en Daedalus 133 (2004), pp. 60-68.

Nussbaum, Martha, "Who is the happy warrior? Philosophy poses questions to psychology", en Eric Posner y Cass Sunstein (editores), Law and Happiness, Chicago, The University of Chicago Press, 2010, pp. 81-113.

PARETo, Vilfredo, Manuale di economía politica, con una introduzione a la scienza sociale, Roma, Societa Editrice Libreria, 1906.

Russell, Bertrand, La conquista de la felicidad, Barcelona, Debolsillo, 2012.

Urzua, Alfonso y Cagueo-Urizar, Alejandra, "Calidad de vida: una revisión teórica del concepto”, en Terapia Psicológica XXX, 1 (2012), p. 61-71.

VAtтimo, Gianni, Más allá del sujeto, Barcelona, Paidós, 1992.

VIGo, Alejandro, La concepción aristotélica de la felicidad, Santiago, Universidad de Los Andes, 1997.

WeINBERgER, Otta, Norm und Institution. Eine Einführung in die Theorie des Rechts, Maguncia, Manz Verlag Viena, 1988.

Wilson, Timothy D. y Gilbert, Daniel T. "Affective forecasting”, en Advances in Experimental Social Psychology 35 (2003), pp. 345-411. 